\title{
ANÁLISIS DE SENSIBILIDAD GLOBAL DEL MODELO DE CULTIVOS SUCROS APLICADO A TOMATE DE CÁSCARA
}

\author{
GLOBAL SENSITIVITY ANALYSIS OF CROP GROWTH SUCROS MODEL APPLIED \\ TO HUSK TOMATO
}

\author{
Irineo L. López-Cruz ${ }^{1 *}$, Abraham Rojano-Aguilar ${ }^{1}$, Raquel Salazar-Moreno ${ }^{1}$ y Rutilo López-López ${ }^{2}$
}

\begin{abstract}
${ }^{1}$ Postgrado de Ingeniería Agrícola y Uso Integral del Agua, Universidad Autónoma Chapingo. Km. 38.5 Carr. México-Texcoco, 56230. Chapingo. Edo. de México. Tel. y Fax: 01 (595) 9521551. 'Instituto de Investigaciones Forestales, Agrícolas y Pecuarias. Km. 1 Carr. Huimanguillo-Cárdenas. 86400, Huimanguillo, Tabasco, México.

*Autor para correspondencia (ilopez@correo.chapingo.mx)
\end{abstract}

\section{RESUMEN}

Dado que el tomate de cáscara (Physalis ixocarpa Brot.) es relevante para la horticultura mexicana pues ocupa el cuarto lugar de la superficie cultivada de hortalizas, se aplicó el modelo SUCROS (a Simple and Universal Crop Growth Simulator) para simular crecimiento potencial de cultivos, en la ejecución de un análisis de sensibilidad y una calibración. Se llevó a cabo un experimento de crecimiento para cuantificar los cambios en biomasa y área foliar del cultivo. Para identificar a los parámetros de entrada del modelo que afectan más las predicciones de las variables respuesta peso seco total, foliar y de frutos, se realizó un análisis de sensibilidad global. Se definieron funciones de densidad uniformes para los parámetros y se usaron la prueba extendida de sensibilidad de amplitud de Fourier y el método de Sobol para calcular los índices de sensibilidad de primer orden y de efectos totales, al inicio, a la mitad y al final del periodo de cultivo. Se ejecutaron 5000 simulaciones Monte Carlo para el primer método y 2700 para el segundo. El modelo fue calibrado mediante el procedimiento de mínimos cuadrados ponderados mediante el uso de los parámetros para los cuales fue más sensible. Se encontró que los parámetros tasa relativa de crecimiento del área foliar, temperatura base, área foliar específica, tasa de asimilación potencial foliar de $\mathrm{CO}_{2}$, eficiencia de uso de la radiación y coeficiente de extinción de la radiación, afectan en mayor medida el comportamiento del modelo SUCROS aplicado a tomate de cáscara. Se obtuvo un buen ajuste para todo el ciclo del cultivo, ya que los coeficientes de correlación entre las predicciones y mediciones directas fueron $0.99,0.99,0.96,0.89$ y 0.95 para peso seco total, de tallos, de frutos, de hojas e índice de área foliar, respectivamente. El error entre el rendimiento estimado y medido fue de $6.2 \mathrm{t} \mathrm{ha}^{-1}$.

Palabras clave: Physalis ixocarpa, distribución uniforme, métodos basados en la varianza, modelo dinámico, simulación Monte Carlo.

\section{SUMMARY}

Given that the husk tomato crop (Physalis ixocarpa Brot.), is relevant for Mexican horticulture, since is fourth in vegetables acreage, the SUCROS (a Simple and Universal Crop Growth Simulator) model for simulation of potential growth was used to implement a sensitivity analysis and model calibration. A crop growth experiment was carried out in order to quantify changes in biomass and leaf area. To identify which model's parameters affect more the predictions of total dry weight, leaf weight and fruits weight a global sensitivity analysis was performed. Uniform probability density functions were defined for each parameter and the extended Fourier amplitude sensitivity test and Sobol methods were used to calculate first order and total effects indices, at the beginning, at the middle and at the end of the crop cultivation period. For the first method 5000 Monte Carlo Simulations were performed and 2700 for the second. The model was calibrated by means of the weighted least squares procedure using their more sensitive parameters. It was found that parameters: relative growth rate of leaf area, base temperature, specific leaf area, potential $\mathrm{CO}_{2}$ assimilation rate, light use efficiency and radiation extinction coefficient, influence to a greater extent the behavior of the SUCROS model applied to husk tomato crop. The high correlation coefficients $0.99,0.99,0.96,0.89$ and 0.95 to total dry weight, stem dry weight, fruits dry weight and leaf area index, respectively, indicate a good fit of the model for overall cultivation period. The error between the estimated and measured yield was $6.2 \mathrm{t} \mathrm{ha}^{-1}$.

Index words: Physalis ixocarpa, uniform distribution, variance-based method, dynamic model, Monte Carlo simulation.

\section{INTRODUCCIÓN}

El tomate de cáscara (Physalis ixocarpa Brot.) es un cultivo importante en la agricultura mexicana que ocupa el cuarto lugar en superficie entre las hortalizas. En 2012 la superficie cosechada a nivel nacional fue de 41,400 ha con una producción de 595,200 $\mathrm{t}$, un rendimiento de $14.4 \mathrm{t} \mathrm{ha}^{-1}$ y un valor de la producción de 2.4 millones de pesos (OEIDRUS Jalisco-SIAP, 2012). El desarrollo y uso de modelos matemáticos mecanicistas o fisiológicos para crecimiento de cultivos es un enfoque potencialmente útil porque permite, además de incrementar el conocimiento del sistema representado, mejorar su manejo como el riego o la fertilización.

El modelo SUCROS (por sus siglas en inglés, a Simple and Universal Crop Growth Simulator) para simular crecimiento potencial, es un modelo dinámico que predice el crecimiento y desarrollo de cultivos en campo abierto (Goudriaan y vanLaar, 1994; van-Laar et al., 1997). Con base en procesos fisiológicos fundamentales como la fotosíntesis y la respiración del cultivo en condiciones óptimas de agua y nutrientes, SUCROS calcula las variables: estado de desarrollo, índice de 
área foliar, y biomasa de hojas verdes, de hojas secas, de raíces, de tallos y de frutos. La biomasa total es la suma de las biomasas de los órganos de la planta. Las variables de entrada del modelo son la radiación solar global $\left(\mathrm{J} \mathrm{m}^{-2} \mathrm{~d}^{-1}\right)$ y la temperatura del aire $\left({ }^{\circ} \mathrm{C}\right)$.

SUCROS ha inspirado el desarrollo de varios modelos de cultivos no solo para campo abierto sino también para invernaderos (Heuvelink, 1999). Sin embargo, hasta donde se sabe, ningún investigador ha aplicado alguna versión de este modelo al cultivo de tomate de cáscara, ya sea para incrementar el conocimiento del sistema de cultivo o el desarrollo de aplicaciones prácticas como optimización del manejo. De acuerdo con la teoría de sistemas dinámicos, las etapas necesarias de la modelación secuencialmente son: derivación de la estructura del modelo, análisis de sensibilidad e identificabilidad, estimación de parámetros (calibración), evaluación (validación), y análisis de incertidumbre (Ljung y Glad, 1994).

Una etapa relevante en el proceso de modelación de crecimiento de cultivos es el análisis de sensibilidad (Cariboni et al., 2007), ya que permite comprender cómo las respuestas del modelo varían en función de los datos de entrada (Saltelli et al., 2000). Recientemente se han desarrollado varios enfoques de análisis de sensibilidad que no utilizan derivadas parciales, tales como coeficientes de regresión estandarizados, gráficas de dispersión, prueba de los efectos elementales o método de Morris, métodos basados en el cálculo de la varianza y filtrado de Monte Carlo (Saltelli et al., 2000; Saltelli et al., 2004; Saltelli et al., 2008). Estos métodos usan el enfoque de muestreo de funciones de densidad de probabilidades. Este paradigma es incipiente para el estudio de modelos para cultivos en campo abierto (Richter et al., 2010).

Últimamente, Makowski et al. (2006) usaron el muestreo del hipercubo Latino para definir los valores base de los parámetros del modelo para crecimiento de cultivos AZODYN al mantener fijos todos los parámetros excepto uno; realizaron $N$ simulaciones (winding stairs) para calcular índices de sensibilidad de primer orden y de efectos totales. Otro método para análisis de sensibilidad global que usaron fue la prueba extendida de sensibilidad de amplitud de Fourier (eFAST). Lamboni et al. (2009) aplicaron un análisis de sensibilidad multivariado a dos modelos de crecimiento de cultivos, y Confalonieri (2010) usó el método de Morris basado en simulación Monte Carlo para estudiar el comportamiento de los modelos para cultivos WOFOST y CropSyst. Más aún, DeJonge et al. (2012) llevaron a cabo un análisis de sensibilidad global para los parámetros del modelo CERES-Maize mediante el uso del método de Morris y del método de Sobol.
A pesar de que existen varios métodos de análisis de sensibilidad global, dos enfoques ampliamente usados por su confiabilidad para identificar los parámetros que más afectan el comportamiento del modelo, son el método eFAST y el método de Sobol (Cariboni et al., 2007; Saltelli et al., 2008; López-Cruz et al., 2012). De acuerdo con lo anterior, los objetivos del presente trabajo fueron: 1) Llevar a cabo un análisis de sensibilidad global para los parámetros del modelo SUCROS para simular crecimiento potencial de cultivos aplicado a un cultivo de tomate de cáscara, con los métodos eFAST y Sobol; y 2) Calibrar el modelo SUCROS, mediante la estimación de los parámetros que el análisis de sensibilidad reportó como los más importantes, a partir de mediciones de las variables peso seco total, de tallos, de frutos, de hojas e índice de área foliar, en un cultivo de tomate de cáscara.

\section{MATERIALES Y MÉTODOS}

\section{Descripción del experimento}

Se estableció un cultivo de tomate de cáscara en una superficie de 1.2 ha con riego por goteo durante el periodo de febrero a junio de 2007 en Chapingo, México, sitio localizado en las coordenadas $19^{\circ} 16^{\prime} 52^{\prime \prime} \mathrm{N}$ y $99^{\circ} 39^{\prime} 0^{\prime \prime} \mathrm{O}$ (López-López et al., 2009). El clima es templado con lluvias en verano y periodo seco durante el invierno, con temperatura media anual de $15.5^{\circ} \mathrm{C}$ y lluvia anual de $664 \mathrm{~mm}$. La variedad de tomate de cáscara fue 'CHF1-Chapingo' obtenida por mejoradores de plantas de la Universidad Autónoma Chapingo.

La fecha de trasplante fue el 30 de marzo de 2007 (día Juliano 89), con una densidad de 16,122 plantas ha ${ }^{-1}$. Para garantizar las condiciones de crecimiento potencial del cultivo, la lámina de riego aplicada se calculó con base en 100 $\%$ de la evapotranspiración de referencia $\left(\mathrm{ET}_{0}\right)$ obtenida mediante el método de Penman-Monteith. En la Figura 1 se muestra la frecuencia de riego aplicada, así como la lluvia registrada durante el periodo del experimento.

La fórmula de fertilización semanal utilizada fue 200N-110P-50K, la cual es suficiente para evitar limitaciones al cultivo de suministro de nitrógeno y fósforo (Pérez y Granados, 2001). Una estación climática GroWheater $\AA$ (Davis Instruments, USA) fue usada para el registro diario de las variables meteorológicas radiación solar global y las temperaturas máxima y mínima (Figura 2). Para determinar la biomasa de los diferentes órganos de la planta y el índice de área foliar, se cosecharon de 3 a 10 plantas seleccionadas aleatoriamente a partir de la fecha del trasplante (días Julianos 103, 117, 124, 138, 145, 159, 166 y 177). Se separaron sus diferentes órganos (raíces, tallos, hojas y frutos), y de cada uno se registró su peso fresco 


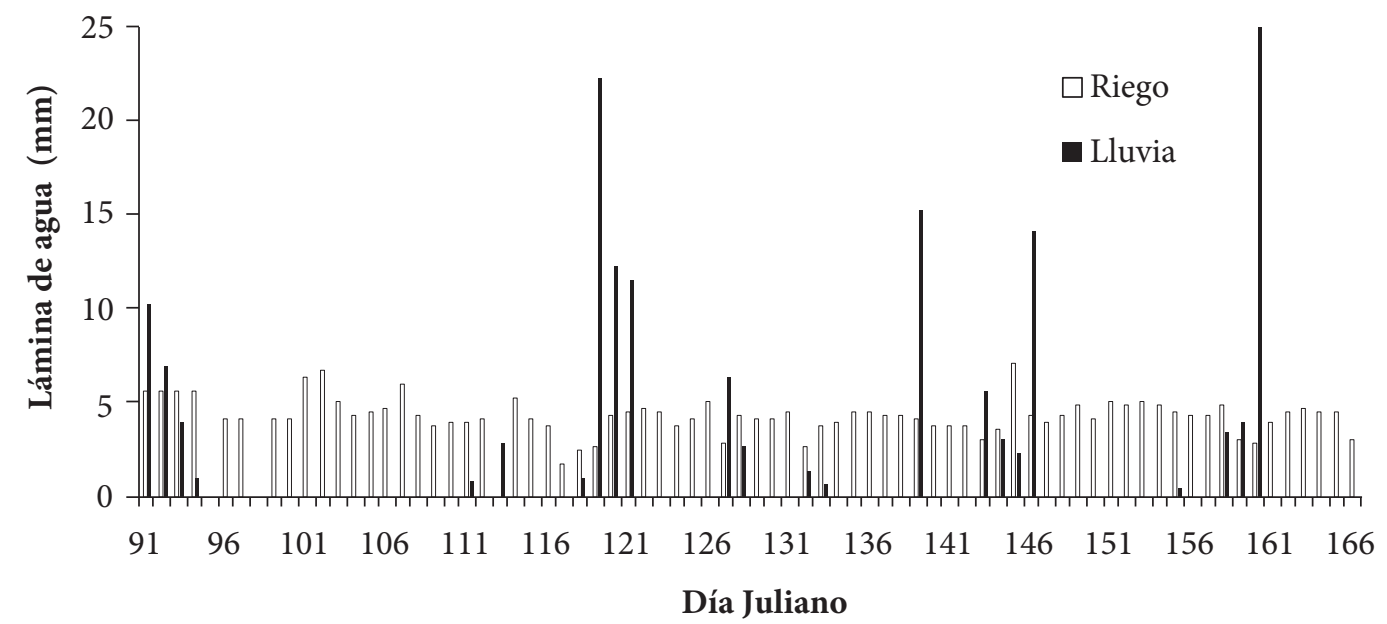

Figura 1. Láminas de riego aplicadas al cultivo de tomate de cáscara, calculadas con base en la evapotranspiración de referencia y la precipitación.

con una balanza electrónica SP2001 (OHAUS® Corp. Pine Brook, NJ, USA), con precisión de 0.1 g. Se midió el área foliar con un integrador de área LICOR LI-3100® (LICOR, INC. Lincoln, NE, USA). Posteriormente las muestras fueron secadas a una temperatura de $75-80{ }^{\circ} \mathrm{C}$ durante 1 a $3 \mathrm{~d}$, variación que dependió de la edad de la planta, y después el peso seco de todos los órganos se midió con una balanza analítica Adventurer Pro (OHAUS® Corp. Pine Brook, NJ, USA), con precisión de $0.0001 \mathrm{~g}$.

\section{El modelo SUCROS para crecimiento potencial de cultivos y su calibración}

La Figura 3 presenta un diagrama relacional del modelo SUCROS para simular crecimiento potencial, cuyas variables de estado son: estado de desarrollo ( $x_{\text {edes }}$, adimensional), índice de área foliar $\left(x_{i a \rho} m^{2} m^{-2}\right)$, biomasa de raíces $\left(x_{\text {raiz }} g m^{-2}\right)$, hojas verdes $\left(x_{h \nu} g m^{-2}\right)$, hojas secas $\left(x_{h s} g m^{-2}\right)$, tallos $\left(x_{\text {tallo }}, g m^{-2}\right)$ y frutos $\left(x_{\text {org }}, g m^{-2}\right)$. La tasa de cambio de la biomasa total se considera como una función de la radiación solar, la temperatura y las características del cultivo. La tasa de asimilación diaria de dióxido de carbono por el follaje se calcula a partir de la cantidad de radiación que es interceptada por el cultivo, la cual depende de la cantidad de radiación entrante y el área foliar del cultivo.

Parte de los carbohidratos producidos son usados en respiración de mantenimiento. El resto se usa para formar nueva biomasa estructural, biomasa que forma paredes celulares y otros componentes celulares (crecimiento). Es decir, en el proceso de conversión una parte de asimilados se consume en respiración de crecimiento. La biomasa producida es dividida entre los diferentes órganos de la planta, mediante coeficientes que dependen del estado fenológico de desarrollo del cultivo. Todos los parámetros del modelo aparecen descritos en el Cuadro 1.

Una vez identificados los parámetros más importantes para el peso seco total, de frutos y de las hojas, mediante el análisis de sensibilidad global, el modelo fue calibrado con el procedimiento de mínimos cuadrados ponderados para hacer equivalentes los valores de peso seco e índice de área foliar. La función a minimizar se definió como la suma de las diferencias al cuadrado entre las variables de respuesta predichas y medidas. Estás fueron: biomasa de tallos, frutos, hojas y total, así como el índice de área foliar. La calidad del ajuste fue medida con la raíz cuadrada relativa del error cuadrático medio (RRECM), la eficiencia de modelación (EF) y el coeficiente de correlación (Wallach et al., 2014). Solo las mediciones realizadas durante los días julianos 103, 117, 124 ,145, 159 y 177 fueron usadas durante la calibración del modelo, dado que se observaron valores atípicos para los días julianos 138 y 166.

\section{Procedimiento del análisis de sensibilidad global}

De acuerdo con Saltelli et al. (2008), un análisis de sensibilidad global consta de las siguientes etapas:

Paso 1. Objetivos del análisis. Determinar los parámetros del modelo SUCROS que tienen una influencia pequeña o grande sobre las predicciones del peso seco, total, foliar y de los frutos de un cultivo de tomate de cáscara, mediante el uso de índices de sensibilidad global basados en las varianzas de las variables de respuesta.

Paso 2. Selección de factores a analizar. En este caso se incluyeron todos los parámetros del modelo SUCROS para 

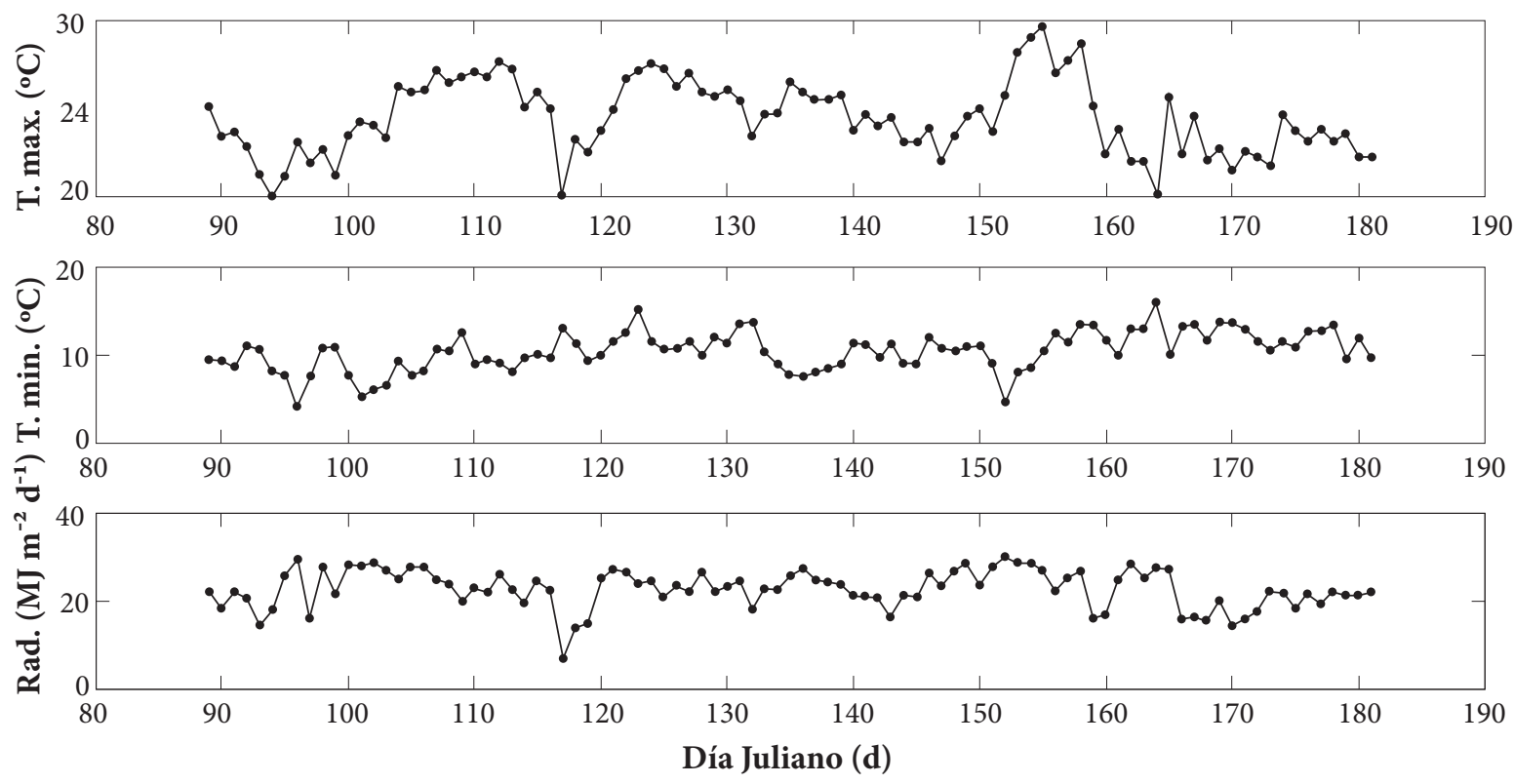

Figura 2. Variables meteorológicas diarias medidas en Chapingo, México, durante el verano de 2007, usadas como variables de entrada del modelo SUCROS.

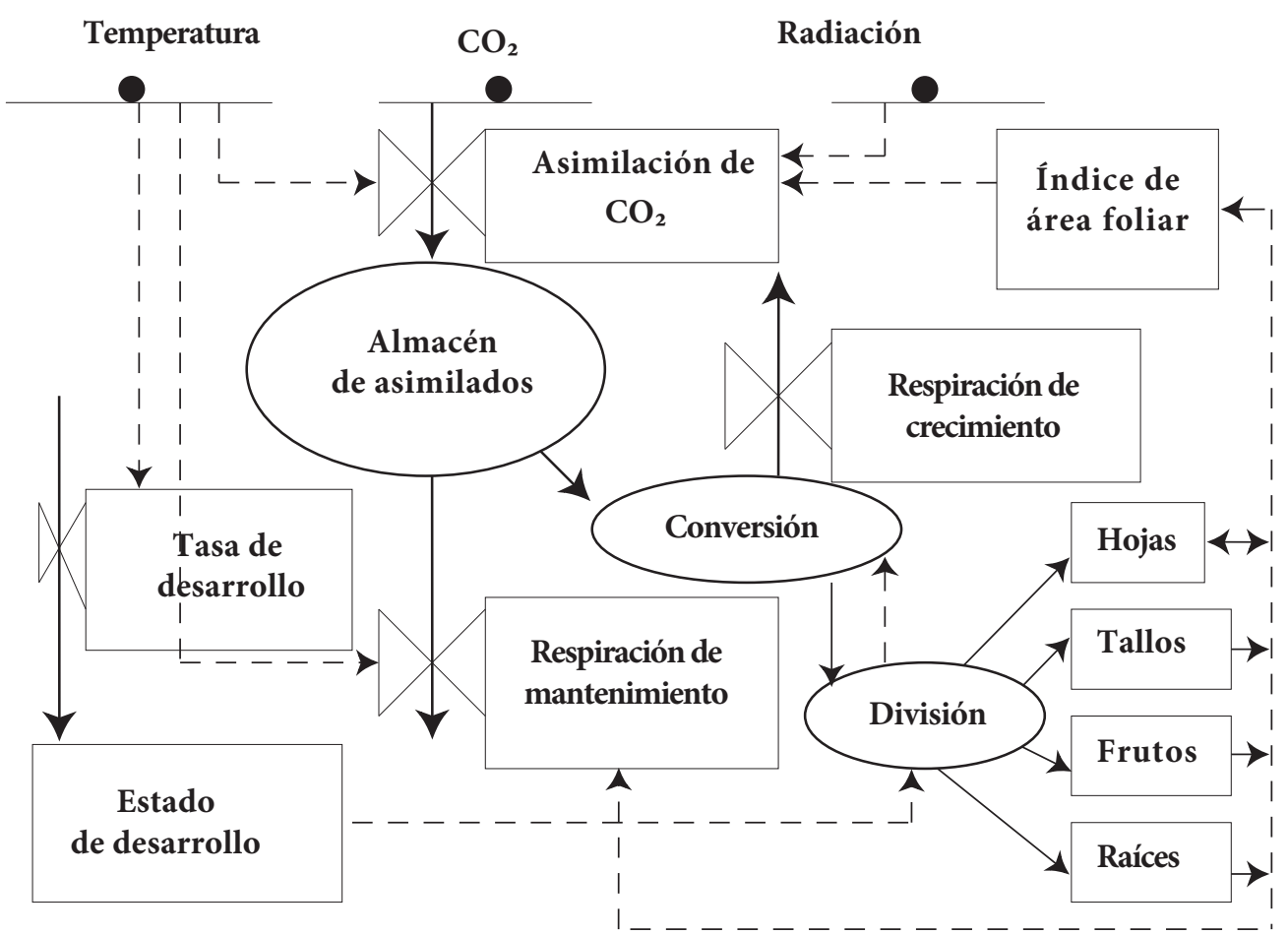

Figura 3. Diagrama relacional del modelo SUCROS para crecimiento potencial de cultivos (van-Laar et al., 1997). 
crecimiento potencial de cultivos (Cuadro 1). Los valores nominales para cultivos anuales fueron tomados de vanLaar et al. (1997). Dado que no existe conocimiento que permita definir el intervalo de variación para cada parámetro, se evaluaron dos niveles de variación alrededor de los valores nominales.

Paso 3. Selección de las funciones de densidad de probabilidades. Se asumió una distribución uniforme para todos los parámetros del modelo SUCROS.

Paso 4. Selección del método de análisis de sensibilidad global. Dado que los métodos que utilizan la varianza de las variables de salida permiten detectar con mayor precisión los parámetros más influyentes en el modelo (Saltelli et al., 2008), se usaron los métodos eFAST y de Sobol.
En ambos es posible calcular un índice de sensibilidad de primer orden y un índice de sensibilidad de efectos totales. El índice de sensibilidad de primer orden $\left(S_{i}\right)$ representa la contribución del efecto principal de cada factor de entrada con respecto a la varianza de la variable de salida:

$$
S_{i}=\frac{V\left[E\left(Y \mid X_{i}\right)\right]}{V(Y)}
$$

donde $V\left[E\left(Y \mid X_{i}\right)\right]$ es la cantidad de varianza esperada (efecto principal) que podría ser removida de la varianza total de la salida $V(Y)$, si se pudiera determinar el verdadero valor del factor $X_{i}$. La varianza total incondicional $(V(Y))$ de la variable de salida $Y$, está dada por:

$$
V(Y)=E\left[V\left(Y \mid X_{i}\right)\right]+V\left[E\left(Y \mid X_{i}\right)\right]
$$

\begin{tabular}{|c|c|c|c|c|}
\hline \multicolumn{2}{|r|}{ Parámetro y número identificador } & \multirow{2}{*}{$\begin{array}{c}\text { Símbolo } \\
\text { RGRL }\end{array}$} & \multirow{2}{*}{$\begin{array}{l}\text { Unidad } \\
\left({ }^{\circ} \mathrm{Cd}\right)^{-1}\end{array}$} & \multirow{2}{*}{$\begin{array}{c}\text { Valor nominal } \\
0.0294\end{array}$} \\
\hline 1 & Tasa relativa de crecimiento del área foliar & & & \\
\hline 2 & Temperatura base para crecimiento área foliar & TBASE & ${ }^{\circ} \mathrm{C}$ & 10.00 \\
\hline 3 & Área foliar específica & SLA & $m^{2} g^{-1}$ & 0.022 \\
\hline 4 & Asimilación foliar $\mathrm{CO}_{2}$ a saturación de luz & AMX & $g m^{2} s^{-1}$ & 0.0011 \\
\hline 5 & Factor de conversión de luz para hojas & EFF & $g j^{-1}$ & $1.25 \times 10^{-6}$ \\
\hline 6 & Coeficiente de extinción para las hojas & $\mathrm{KDF}$ & $m^{2} m^{-2}$ & 0.650 \\
\hline 7 & Coeficiente de dispersión foliar para PAR & SCP & Adimensional & 0.200 \\
\hline 8 & Factor aumento respiración de mantenimiento & Q10 & Adimensional & 2.000 \\
\hline 9 & Temperatura de referencia & TREF & ${ }^{\circ} \mathrm{C}$ & 25.000 \\
\hline 10 & Respiración de mantenimiento foliar & MAINLV & $g g^{-1} d^{-1}$ & 0.030 \\
\hline 11 & Respiración de mantenimiento de tallos & MAINST & $g g^{-1} d^{-1}$ & 0.015 \\
\hline 12 & Respiración de mantenimiento de raíces & MAINRT & $g g^{-1} d^{-1}$ & 0.015 \\
\hline 13 & Respiración de mantenimiento de los frutos & MAINSO & $g g^{-1} d^{-1}$ & 0.010 \\
\hline 14 & Requerimiento asimilados de biomasa foliar & ASRQLV & $g g^{-1}$ & 1.463 \\
\hline 15 & Requerimiento asimilados de biomasa tallos & ASRQST & $g g^{1}$ & 1.513 \\
\hline 16 & Requerimiento asimilados para biomasa raíces & ASRQRT & $g g^{1}$ & 1.444 \\
\hline 17 & Requerimiento asimilados para biomasa frutos & ASRQSO & $g g^{1}$ & 1.490 \\
\hline 18 & Índice de área foliar crítico & LAICR & $m^{2} m^{-2}$ & 4.000 \\
\hline 19 & Fracción biomasa de tallos translocado a frutos & FRTRL & Adimensional & 0.200 \\
\hline 20 & Fracción de masa de carbón foliar & CFLV & $g g^{-1}$ & 0.459 \\
\hline 21 & Fracción de masa de carbón de tallos & CFST & $g g^{-1}$ & 0.494 \\
\hline 22 & Fracción de masa de carbón de raíces & CFRT & $g g^{1}$ & 0.467 \\
\hline 23 & Fracción de masa de carbón en frutos & CFSO & $g g^{-1}$ & 0.471 \\
\hline 24 & Incremento de tasa relativa de mortalidad foliar & FRDR & Adimensional & 1.000 \\
\hline & $\begin{array}{l}\text { Factor de conversión para removilización de } \mathrm{CHO} \text { de } \\
\text { tallos en glucosa }\end{array}$ & CONVL & Adimensional & 0.947 \\
\hline
\end{tabular}


donde $E\left[V\left(Y \mid X_{i}\right)\right]$ es el valor esperado de la varianza de la variable respuesta de salida que podría permanecer inexplicada (varianza residual), si $X_{i}$ fuera variado libremente sobre su rango de incertidumbre. Para el presente estudio $i=1, \ldots, 25$ ya que se estudiaron 25 parámetros del modelo SUCROS. Este índice cumple la condición $0 \leq S_{i} \leq 1$ (Saltelli et al., 2008). El índice de efectos totales $\left(S_{T_{i}}\right)$ da cuenta de la contribución total de la salida debida al factor $X_{i}$; es decir, los efectos de primer orden más los efectos debido a las interacciones entre los parámetros. Al reescribir la Ec. 2 condicionada respecto a todos los factores excepto $X_{\sim i}$ se obtiene:

$$
\begin{gathered}
V(Y)=E\left[V\left(Y \mid X_{\sim i}\right)\right]+V\left[E\left(Y \mid X_{\sim i}\right)\right] \\
\left.\left.S_{T_{i}}=1-\frac{V\left[E\left(Y \mid X_{\sim i}\right)\right.}{V(Y)}\right]=\frac{E\left[V\left(Y \mid X_{\sim i}\right)\right.}{V(Y)}\right]
\end{gathered}
$$

donde $V\left[E\left(Y \mid X_{\sim i}\right)\right]$ es la cantidad esperada de varianza que sería removida de la varianza total al considerar todos los términos de cualquier orden que incluyan el factor $X_{i}$. En general, $S_{T_{i}} \geq S_{i}$ (Saltelli et al., 2008). Para calcular los índices de sensibilidad global se siguió el procedimiento numérico propuesto por Saltelli et al. (2008), implementado en el programa de computadora para análisis de sensibilidad para el ambiente de programación Matlab.

La interpretación de los índices, de acuerdo con los mismos autores, es como sigue: si el factor $X_{i}$ no está involucrado en las interacciones con otros factores, entonces $S_{T_{i}}=$ $S_{i}$ de lo contrario $S_{T_{i}}>S_{i}$. La diferencia $S_{T_{i}}-S_{i}$ indica en qué medida el factor $X_{i}$ está involucrado en las interacciones. Si se obtiene $S_{T_{i}}=0$ implica que el factor $X_{i}$ no es influyente y puede ser fijado en cualquier valor, sin afectar la varianza de la variable de salida. Además, la suma de todos los $S_{i}$ es igual a la unidad en el caso de modelos aditivos, y menor que uno en caso de modelos no aditivos. La suma de todos los $S_{T_{i}}$ es siempre mayor a uno, y solamente es igual a la unidad en el caso de modelos aditivos.

Paso 5. Generación de la muestra de entrada. Una muestra de tamaño $\mathrm{N}=5000$ se generó en el caso de método eFAST, y una de tamaño $\mathrm{N}=2700$ en caso del método de Sobol, para garantizar una buena estimación de los índices de sensibilidad como se recomienda en la simulación Monte Carlo (Saltelli et al., 2004). Se observó que una muestra más grande en el caso del método de Sobol provocaba un error en el algoritmo.

Paso 6. Simulaciones con el modelo SUCROS. Se usó simulación Monte Carlo con los valores de los parámetros del modelo obtenidos de las muestras generadas en el Paso 5. Se alimentó el modelo SUCROS con estos valores, para ejecutar 5000 y 2700 simulaciones, respectivamente. Se re- gistraron los valores que predice el modelo para las variables biomasa de frutos, biomasa foliar (hojas verdes y secas) y peso seco total (hojas, raíces, tallos y frutos), en tres diferentes tiempos del periodo de crecimiento del cultivo (20, 40 y 74 d después de la emergencia).

\section{RESULTADOS Y DISCUSIÓN}

\section{Índices de sensibilidad calculados al final del periodo de crecimiento}

De acuerdo con el método eFAST (Figura 4), solamente siete parámetros, a saber: RGRL, TBASE, SLA, AMX, EFF, KDF y ASRQSO, son los más importantes en el modelo SUCROS para simular el crecimiento potencial del cultivo de tomate de cáscara. Esto se observó para la biomasa de hojas, de frutos y de toda la planta. Para el subconjunto de parámetros más influyentes se cumple la condición $S_{T_{i}}>S_{i}$ lo cual significa que todos los parámetros participan en interacciones con otros parámetros. Dado que se obtuvieron valores $S_{T_{i}}-S_{i}$ del parámetro AMX (0.0091 [0.01], 0.0093 [0.09], 0.0087 [0.15], para peso seco total, frutos y hojas, respectivamente) y de TBASE (0.0087 [0.06], 0.0081 [0.06], 0.0131 [0.06] para peso seco total, frutos, y hojas), significa que estos dos parámetros están más involucrados en las interacciones con otros parámetros. Los valores relativos $\left(S_{T_{i}}-S_{i}\right) / S_{T_{i}}$ se muestran entre paréntesis cuadrados.

Puesto que $\Sigma S_{T_{i}}>1(1.19,1.20$ y 1.24 , para biomasa total, de frutos y de hojas), el modelo es altamente no aditivo. El conjunto de parámetros con índice de primer orden $S_{i}=0 \mathrm{e}$ índice de efectos totales $S_{T_{i}}$ cercano a cero, está formado por SCP, MAINRT, LAICR, FRTRL, CFLV, CFST, CFRT, CFSO, FRDR y CONVL, y sus valores se consideran asociados con biomasa total, frutos y hojas. En el caso del peso seco foliar también MAINSO resultó ser no relevante.

Con el método de Sobol se obtuvieron resultados similares a los observados con el procedimiento eFAST, como puede apreciarse en la Figura 5. Pero en contraste con eFAST, ASRQSO resultó ser un parámetro importante. Los parámetros menos importantes fueron los mismos que para eFAST, con excepción de MAINSO. Pero a diferencia de eFAST, en el caso de Sobol se obtuvo exactamente un índice de efectos totales $S_{T_{i}}=0$ para los parámetros no influyentes en el modelo. Esto puede observarse al comparar las Figuras 4 y 5 .

El análisis anterior se llevó a cabo con un intervalo de incertidumbre para todos parámetros del modelo SUCROS de $\pm 10 \%$ alrededor de los valores nominales. Sin embargo, los resultados fueron prácticamente los mismos con intervalos de incertidumbre generados con $\pm 20 \%$ de variación alrededor de los valores nominales. Este comportamiento puede 

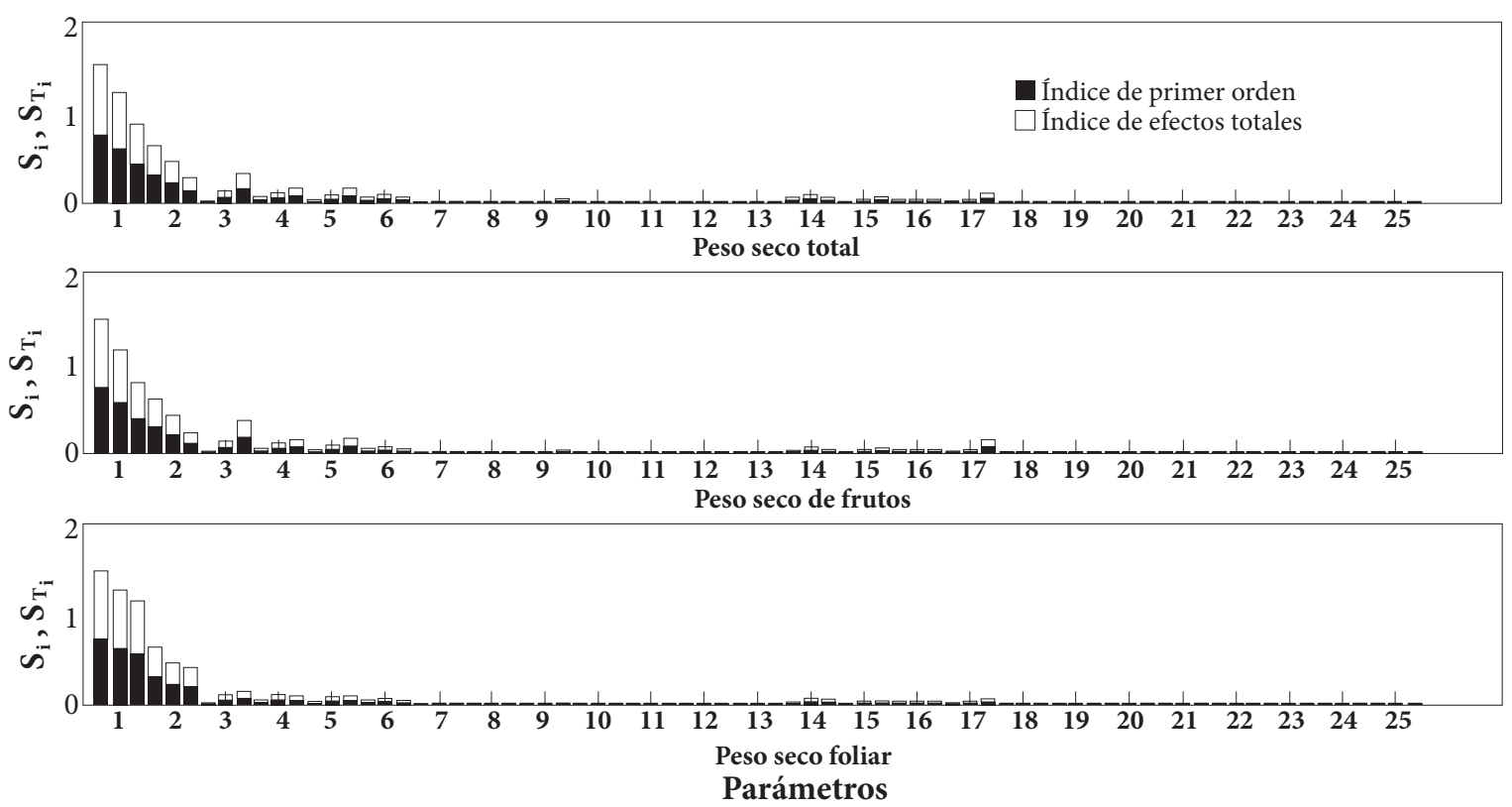

Figura 4. Índices de sensibilidad global calculados mediante el método eFAST para los parámetros del modelo SUCROS aplicado al cultivo de tomate de cáscara, a 20, 40 y 74 días después de emergencia, con un intervalo de incertidumbre de $10 \%$.
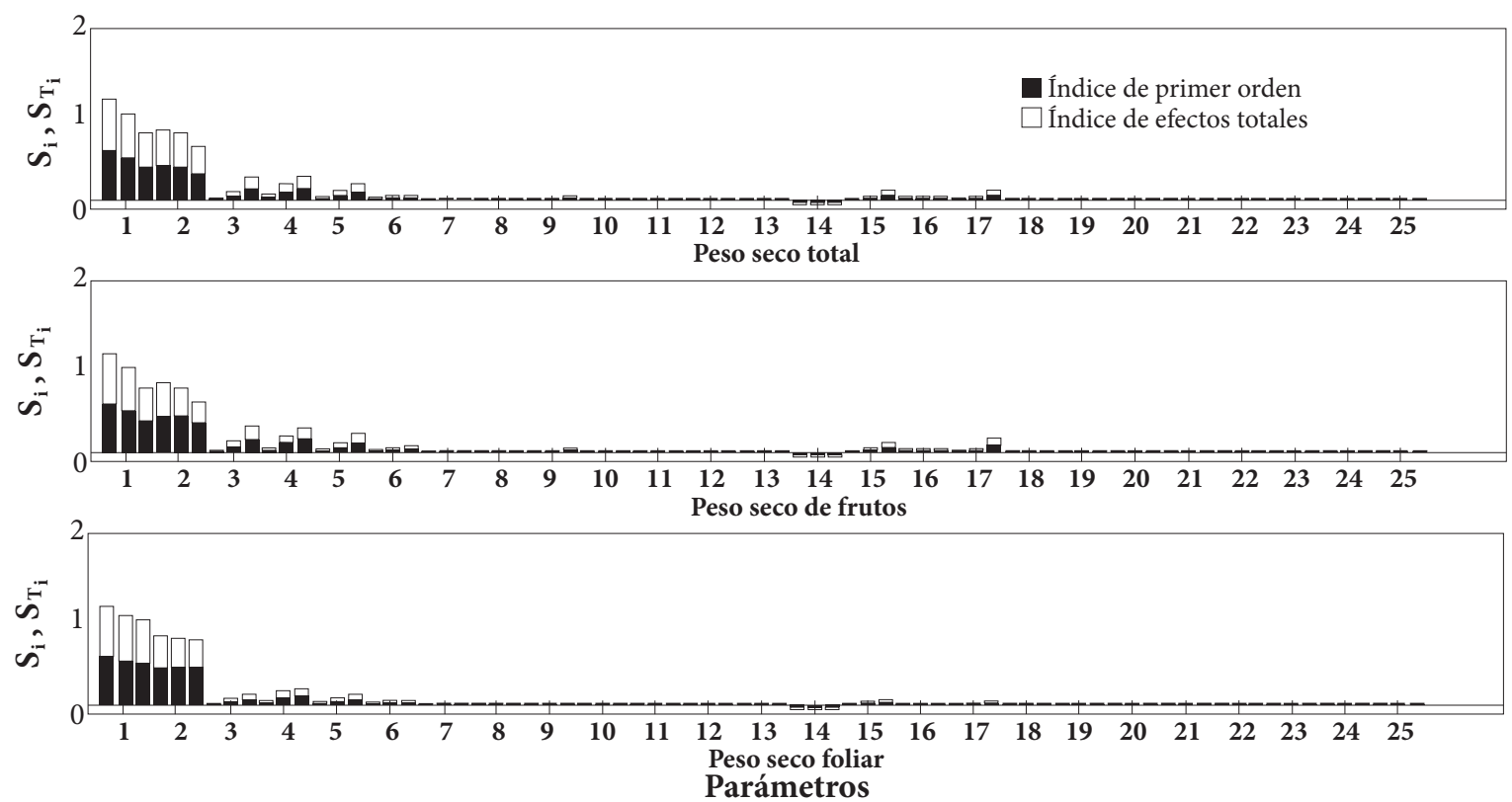

Figura 5. Índices de sensibilidad global obtenidos mediante el método de Sobol para los parámetros del modelo SUCROS aplicado a un cultivo de tomate de cáscara, a 20, 40 y 74 días después de emergencia, con un intervalo de incertidumbre de $10 \%$.

observarse en la Figura 6 solamente para el caso del método eFAST.

\section{Índices de sensibilidad calculados durante el periodo de crecimiento}

En contraste con los índices de sensibilidad calculados al final del periodo de crecimiento, al principio solamente
RGRL y TBASE afectan el comportamiento del modelo SUCROS aplicado al tomate de cáscara. Esto puede apreciarse en la Figura 4 para las tres variables: biomasa total, de los frutos y de las hojas. Para estos parámetros se observó que $S_{T_{i}}>S_{i}$ lo cual significa que toman parte en las interacciones. De hecho, las mayores diferencias $S_{T_{i}}-S_{i}$ entre los índices se observaron para los parámetros RGRL y TBASE, y fueron de 0.0143 [0.01] y 0.0189 [0.05] en el caso del peso 

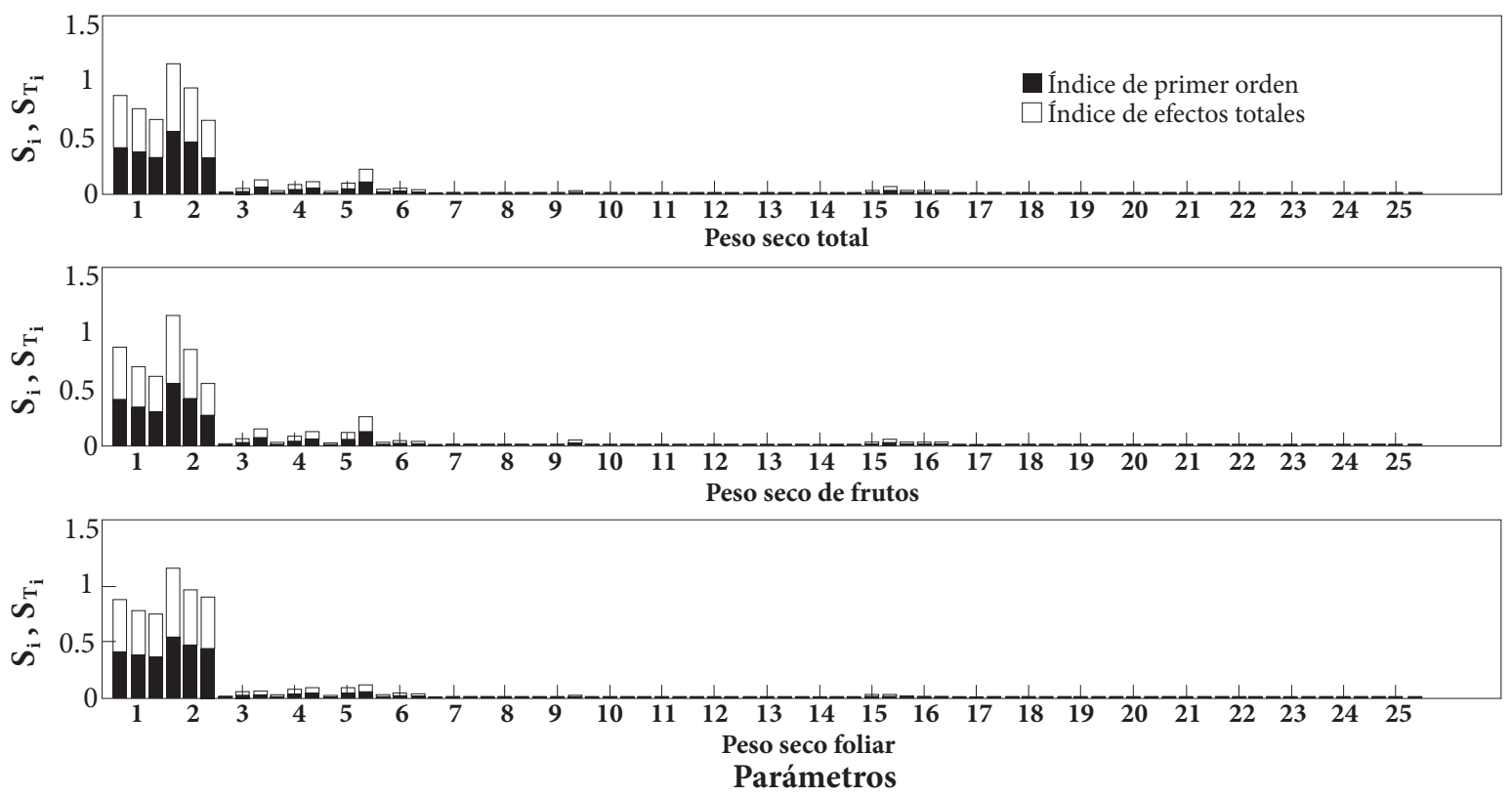

Figura 6. Índices de sensibilidad global obtenidos mediante el método eFAST para los parámetros del modelo SUCROS aplicado a un cultivo de tomate cáscara, a 20, 40 y 74 días después de emergencia, con un intervalo de incertidumbre de $20 \%$.

seco total, de 0.0149 [0.01] y 0.0201 [0.05] en el caso de peso seco de frutos, y de 0.0144 [0.02] y 0.0193 [0.05] para peso seco de las hojas. Las diferencias relativas con respecto a $S_{T_{i}}$ están entre paréntesis cuadrados.

Una indicación de que el modelo no es aditivo fue que $\Sigma S_{T_{i}}>S_{i}(1.34,1.32$ y 1.34 , respectivamente). Además, los parámetros que no afectan el comportamiento del modelo SUCROS y que presentaron un índice de primer orden $\left(S_{i}=\right.$ 0 ) fueron más que los correspondientes al final del periodo de crecimiento. El conjunto de estos parámetros incluyó a SLA, MAINST y MAINSO para peso seco total y peso seco foliar, y para peso seco de frutos fueron SLA, MAINLV, MAINST y MAINSO.

Al comparar las Figuras 4 y 5 se puede observar que ambos métodos arrojaron los mismos resultados. Aunque para el método Sobol el conjunto de los parámetros con índices de efectos totales $S_{T_{i}}=0$ incluyó además a los coeficientes SLA, MAINLV, MAINST y MAINSO. Esto fue observado para las tres variables: biomasa total, biomasa de frutos y biomasa de hojas. El comportamiento de los índices de sensibilidad a la mitad del periodo de crecimiento se muestra en la Figura 4 para el método eFAST, y en la Figura 5 para el método Sobol. De acuerdo con ambos métodos, los parámetros más importantes resultaron ser RGRL, TBASE, SLA, AMX, EFF y KDF, para las tres variables estudiadas.

Con el método eFAST se obtuvo $S_{T_{i}}>S_{i}$ para los parámetros más influyentes, lo cual significa que todos ellos participan en interacciones con otros parámetros. Las diferencias
$S_{T_{i}}-S_{i}$ fueron: 0.0102 [0.01], 0.0147 [0.06], 0.0070 [0.10], 0.0072 [0.11], 0.0087 [0.17], y 0.0073 [0.20], respectivamente para la biomasa total; 0.0098 [0.01], 0.0140 [0.06], 0.0073 [0.08], 0.0072 [0.11], 0.0089 [0.16] y 0.0074 [0.19] para la biomasa de frutos; y 0.0108 [0.01], 0.0159 [0.06], 0.0068 [0.12], 0.0071 [0.12], 0.0084 [0.19] y 0.0073 [0.20] para biomasa de hojas. Dado que $\Sigma S_{T_{i}}>S_{i}$ para el peso seco total, de frutos y foliar $(1.26,1.24$, y 1.27 , respectivamente), se puede esperar que el modelo no sea aditivo.

Los parámetros que no afectan el comportamiento del modelo, con índice de primer orden de acuerdo con el método eFAST, fueron los mismos que en el caso de los índices calculados al final del periodo de crecimiento, con excepción de los coeficientes SCP, MAINST y MAINSO. Este comportamiento se observó para las tres variables predichas por el modelo. Con el método de Sobol los parámetros no influyentes fueron los mismos que en el caso del método eFAST. Como en el caso de las sensibilidades al final del periodo de crecimiento, los resultados fueron los mismos al usar intervalos de incertidumbre con $\pm 10 \%$ y $\pm 20 \%$ de variación alrededor de los valores nominales para todos los parámetros.

Con ambos métodos estudiados se encontró que un pequeño subconjunto de parámetros del modelo SUCROS explican la mayor parte de la varianza de las variables peso seco total, de frutos y foliar de un cultivo de tomate de cáscara. Estos resultados son similares a los reportados por Vazquez-Cruz et al. (2014), quienes también usaron el método eFAST y Sobol para estudiar el comportamiento del 
modelo TOMGRO para el cultivo de tomate (Lycopersicon esculentum) crecido en condiciones de invernadero.

\section{Simulaciones y calibración del modelo SUCROS para tomate de cáscara}

La comparación entre las predicciones del modelo SUCROS y las mediciones experimentales muestran un buen ajuste, como se observa en la Figura 7. Los valores de RRECM fueron: $0.10,0.24,0.45,0.41$ y 0.23 , para peso seco total, de tallos, de frutos, de hojas e IAF, respectivamente. Los correspondientes valores del coeficiente de correlación fueron: $0.99,0.99,0.96,0.89$ y 0.95 . Mientras que los valores de la eficiencia de modelación fueron: $0.99,0.93,0.85$, 0.80 y 0.91. Los valores de los parámetros estimados fueron respectivamente: EFF $\left(3.1832 \times 10^{-6}\right)$, SLA (0.0149), KDF (0.98), RGRL (0.0279) y AMX (0.0111), todos considerablemente mayores que los valores nominales.

El subconjunto de parámetros con los que el modelo resultó ser más sensible incluye a la tasa relativa de crecimiento del área foliar (RGRL), la cual es especialmente relevante durante la etapa juvenil del cultivo. El parámetro área foliar específica (SLA) afecta el cálculo de la fotosíntesis, como ha sido analizado por Goudriaan y van-Laar (1994) en el caso de una versión temprana del modelo SUCROS.

En general, un resultado relevante del presente trabajo es que los parámetros relacionados con la intercepción de la radiación y la fotosíntesis foliar y del cultivo, como la efi- ciencia de uso de la luz (EFF), el coeficiente de extinción (KDF) y la tasa de asimilación de $\mathrm{CO}_{2}$ a saturación de luz (AMX), resultaron ser los más influyentes sobre el comportamiento del modelo SUCROS. Estos resultados están de acuerdo con el comportamiento esperado para los modelos de crecimiento de cultivos basados en la fotosíntesis (Goudriaan y van-Laar, 1994). El rendimiento de frutos estimado por el modelo fue de $47.9 \mathrm{t} \mathrm{ha}^{-1} \mathrm{y}$ el medido de $41.7 \mathrm{t} \mathrm{ha}^{-1}$, con un error de predicción de $6.2 \mathrm{t} \mathrm{ha}^{-1}$. El valor predicho es aproximado al promedio de $49.4 \mathrm{t} \mathrm{ha}^{-1}$ reportado por López-López et al. (2009).

\section{CONCLUSIONES}

Dos métodos de análisis de sensibilidad global basado en el cálculo de las varianzas de la variable de respuesta permitieron determinar el subconjunto de parámetros más influyentes del modelo SUCROS para crecimiento potencial aplicado a un cultivo de tomate de cáscara. A pesar de que el modelo tiene 25 parámetros, el análisis de sensibilidad global reportó que los parámetros tasa relativa de crecimiento del área foliar, temperatura base, área foliar específica, tasa de asimilación potencial foliar $\mathrm{CO}_{2}$, eficiencia de uso de la radiación y coeficiente de extinción de la radiación, que intervienen en la intercepción de la radiación y estimación de la fotosíntesis foliar y del cultivo, son los que más influyen en el comportamiento de la biomasa foliar, de los frutos y del total que predice el modelo SUCROS. La calibración del modelo con estos parámetros mostró un buen ajuste, ya que los coeficientes de correlación entre las predicciones y

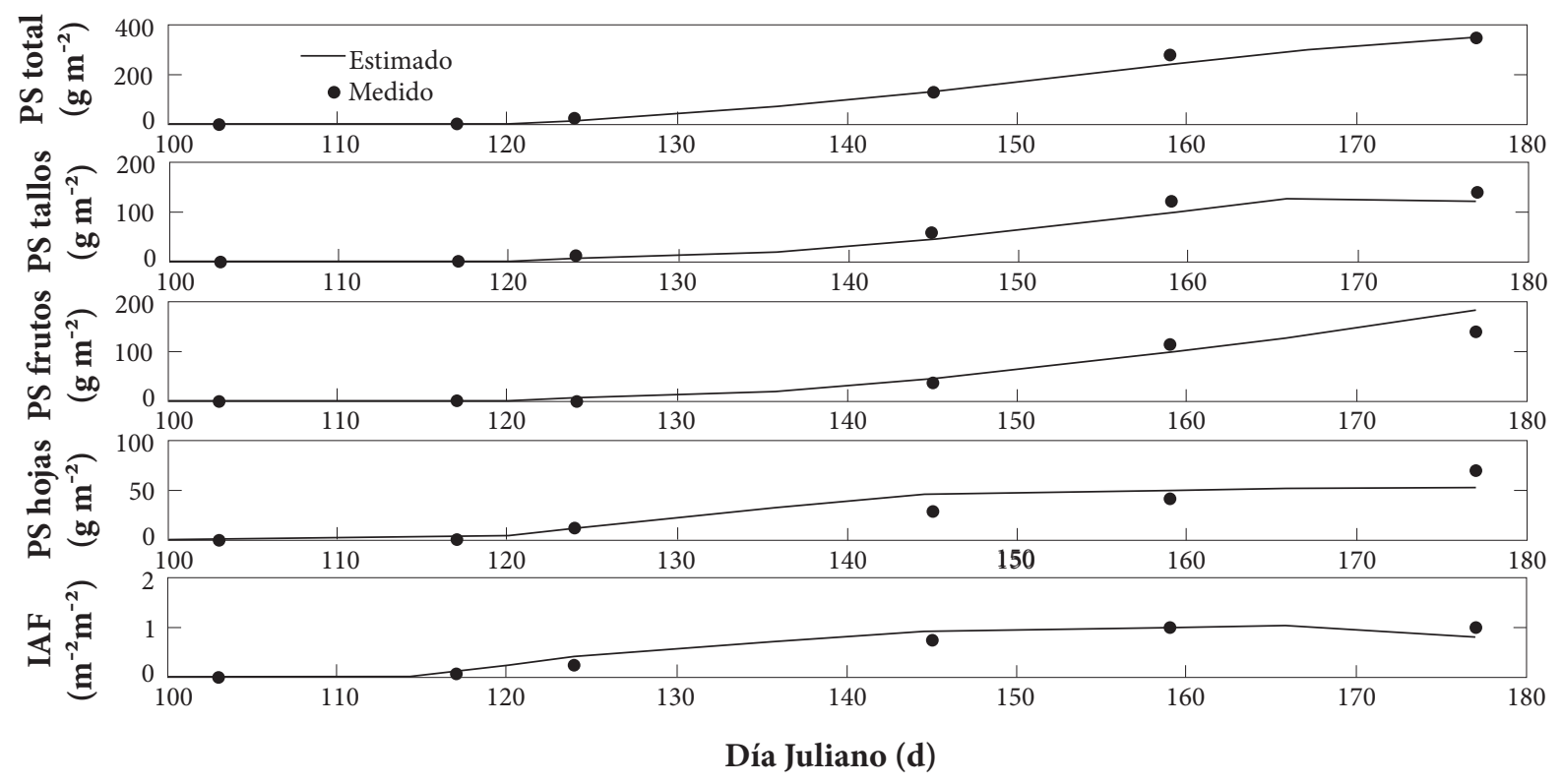

Figura 7. Índice de área foliar y biomasa total, de tallos, de frutos y de hojas predichos por el modelo SUCROS para crecimiento potencial aplicado a un cultivo de tomate de cáscara, después de su calibración, comparadas contra mediciones directas. 
mediciones fueron de $0.99,0.99,0.96,0.89$ y 0.95 para peso seco total, de tallos, de frutos, de hojas e índice de área foliar, respectivamente.

\section{BIBLIOGRAFÍA}

Cariboni J., D. Gatelli, R. Liska and A. Saltelli (2007) The role of sensitivity analysis in ecological modelling. Ecological Modelling 203:167-182

Confalonieri R. (2010) Monte Carlo based sensitivity analysis of two crop simulators and considerations on model balance. European Journal of Agronomy 33:89-93.

DeJonge K. C., J. C. Ascough-II, M. Ahmadi, A. A. Andalles and M. Arabi (2012) Global sensitivity and uncertainty analysis of a dynamic agroecosystem model under different irrigation treatments. Ecological Modelling 231:113-125.

Goudriaan J. and H. H. van-Laar (1994) Modelling Potential Crop Growth Processes. Textbook with exercises. Kluwer Academic Publishers. Dordrecht, The Netherlands. 239 p.

Heuvelink E. (1999) Evaluation of a dynamic simulation model for tomato crop growth and development. Annals of Botany 83:413-422.

Lamboni M., D. Makowski, S. Lehuger, B. Gabrielle and H. Monod (2009) Multivariate global sensitivity analysis for dynamic crop models. Field Crops Research 113:312-320.

Ljung L. and T. Glad (1994) Modeling of Dynamic Systems. Prentice-Hall Englewood Cliffs. New Jersey, USA. 368 p.

López-Cruz I. L., R. Salazar-Moreno, A. Rojano-Aguilar y A. RuízGarcía (2012) Análisis de sensibilidad global de un modelo de lechugas (Lactuca sativa L.) cultivadas en invernadero. Agrociencia $46: 383-397$

López-López R., R. Arteaga-Ramírez, M. A. Vázquez-Peña, I. L. LópezCruz e I. Sánchez-Cohen (2009) Producción de tomate de cáscara (Physalis ixocarpa Brot.) basada en láminas de riego y acolchado plástico. Revista Chapingo Serie Horticultura 15:83-89.
Makowski D., C. Naud, M. H. Jeuffroy, A. Barbottin and H. Monod (2006) Global sensitivity analysis for calculating the contribution of genetic parameters to the variance of crop model prediction. Reliability Engineering and Systems Safety 91:1142 1147.

OEIDRUS-Jalisco-SIAP, Oficina Estatal de Información para el Desarrollo Rural Sustentable, Sistema de Información Agrícola y Pecuaria (2012) http://www.oeidrus-jalisco.gob.mx/ (Marzo 2014)

Pérez M. L. y A. J. Granados (2001) Fertilización nitro-fosfórica en tomate de cáscara (Physalis ixocarpa Brot.) de riego, en Irapuato, Gto., México. Acta Universitaria 11:19-25.

Richter G. M., M. Acutis, P. Trevisiol, K. Latiri and R. Confalonieri (2010) Sensitivity analysis for a complex crop model applied to Durum wheat in the Mediterranean. European Journal of Agronomy 32:127-136

Saltelli A., K. Chan and E. M. Scott (2000) Sensitivity Analysis. John Wiley \& Sons Ltd. Chichester, UK. 475 p.

Saltelli A., S. Tarantola, F. Campolongo and M. Ratto (2004) Sensitivity analysis in practice. A Guide to Assessing Scientific Models. John Wiley \& Sons Ltd. Chichester, UK. 219 p.

Saltelli A., M. Ratto, F. Campolongo, J. Cariboni, D. Gatelli, M. Saisana and S. Tarantola (2008) Global Sensitivity Analysis. The Primer. John Wiley \& Sons Ltd. Chichester, UK. 292 p.

van-Laar H. H., J. Goudriaan and H. van-Keulen (1997) SUCROS97: simulation of crop growth for potential and water-limited production situations, as applied to spring wheat. Quantitative Approaches in Systems Analysis, vol. 14. Ed. AB-DLO. Wageningen, The Netherlands. 78 p.

Vazquez-Cruz M.A., R. Guzman-Cruz, I.L. Lopez-Cruz, O. CornejoPerez, I. Torres-Pacheco and R.G. Guevara-Gonzalez (2014) Global sensitivity analysis by means of EFAST and Sobol' methods and calibration of reduced state-variable TOMGRO model using genetic algorithms. Computers and Electronics in Agriculture 100:1-12.

Wallach D., D. Makowski, J.W. Jones and F. Brun (2014) Working with Dynamic Crop Models. Academic Press. London UK. 487 p. 